\title{
Darboux Frame of a Curve Lying on a Lightlike Surface
}

\author{
E. Selcen Yakıcı Topbaş*, İsmail Gök, Nejat Ekmekci and Yusuf Yaylı
}

(Communicated by Bülent ALTUNKAYA)

\begin{abstract}
In this study, the Darboux frame is given for a curve lying on the lightlike surface of three dimensional Minkowski space. Moreover, known curvatures of the curve are obtained according to the Darboux frame and relationships between these curvatures are acquired. In specific case, by taking into consideration that the surface is a lightlike cone, similar characterizations are investigated.
\end{abstract}

Keywords: Darboux frame; Lightlike surface; Lightlike cone; Helix.

AMS Subject Classification (2010): 14H45, 14H50, 53A04.

*Corresponding author

\section{Introduction}

A surface is called lightlike surface in $\mathbb{E}_{1}^{n}$ if its normal vector is lightlike everywhere (zero length with respect to the Lorentz metric), but the vector is not identically zero. Lightlike cone is a good example of this surface. From the point of view of physics, lightlike surfaces are of importance because they are models of different types of horizons, such as event horizons, Cauchy's horizons, Kruskal horizons, studied in relativity theory. Lightlike submanifolds, hypersurfaces are also studied in the theory of electromagnetism $[1,2,6]$. Due to the degenerate metric of lightlike

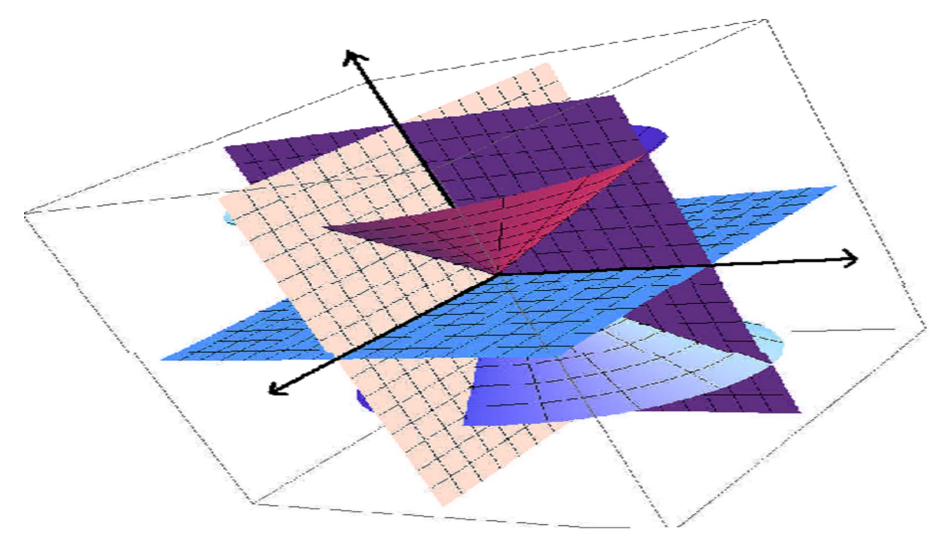

Figure 1. The lightlike cone (an example of lightlike surface).

submanifolds, the usual way of the theory of non-degenerate geometry can not be useful. The primary difference between the lightlike submanifolds and the non-degenerate submanifolds is that in the first case the normal vector bundle intersects the tangent bundle. In other words, a vector of a tangent space $T_{x} \bar{M}$ can not be decomposed

Received : 02-June-2016, Accepted : 18-July-2016 
uniqely into a component tangent to $T_{x} M$ and a component of normal space $T_{x} M^{\perp}$. As a result, the lightlike geometry is quite different from Riemannian and semi- Riemannian geometry.

A pseudo-Riemannian manifold is a differetiable manifold equipped with pseudo-Riemannian metric which is non-degenerate, smooth, symmetric metric tensor. This metric tensor need not be positive definite. The Pseudo (semi)-Euclidean space is denoted by $\mathbb{E}_{v}^{n}$. If $v=0, \mathbb{E}_{v}^{n}$ semi-Euclidean spaces reduce to $\mathbb{E}^{n}$ Euclidean space, that is, semi-Euclidean space is a generalization of Euclidean space. For $v=1$ and $n \geqslant 1, \mathbb{E}_{1}^{n}$ is called Lorentz- Minkowski $n$-space.

Lorentz manifold form the most important subclass of semi-Riemannian manifolds because of their physical application to the theory of relativity. Due to semi-Riemannian metric there are three different kind of curves, namely spacelike, timelike, lightlike(null) depending on the casual character of their tangent vectors, that is, the curve $\alpha$ is called a spacelike (resp. timelike and lightlike) if $\alpha^{\prime}(t)$ is spacelike (resp. timelike and lightlike) for any $t \in I[1,2,6]$.

\section{Darboux Frame On Lightlike Surfaces}

In this section, we give the Darboux frame for curves lying on a lightlike surface of three dimensional Minkowski space (The definition of the Darboux Frame in Euclidean space is inspired us[7]). Moreover, we obtain known curvatures of the curve according to the Darboux frame and give the relationships between these curvatures (relationships between the curvatures $\kappa, \tau$ which are known curvatures in $\mathbb{E}_{1}^{3}$ and $\kappa_{z}, \kappa_{y}, \tau_{r}$ which are Darboux frame's curvature in $\mathbb{E}_{1}^{3}$ ).

Let $M$ be a lightlike surface in $\mathbb{E}_{1}^{3}$ (3-dimensional Minkowski space), $\alpha: I \rightarrow M$ be spacelike curve with arc length parameter $s$ on $M$, and also $Z$ be the normal vector field of that surface $T$ is to be unit tangent vector field of $\alpha$, then there exists a lightlike vector $Y$ satisfiying the conditions provided as follows,

$$
\begin{gathered}
\langle Z, Z\rangle=\langle Y, Y\rangle=\langle Z, T\rangle=\langle Y, T\rangle=0 \\
\langle Z, Y\rangle=\langle T, T\rangle=1 .
\end{gathered}
$$

All $\alpha$ curves considered here are arc length parametrized curves unless otherwise stated.

Definition 2.1. Let $\alpha: I \rightarrow M \subset \mathbb{E}_{1}^{3}, s \mapsto \alpha(s)$ be a spacelike curve on the lightlike surface $M, T$ be tangent vector field of $\alpha$ and $Y, Z$ are the lightlike vectors which are satisfying Eq. (2.1). Then, $\{T, Y, Z\}$ establishes a frame field along the curve $\alpha$ and it is called Darboux frame along the curve $\alpha$ of the lightlike surface $M$.

Definition 2.2. Let $\alpha: I \rightarrow M \subset \mathbb{E}_{1}^{3}, s \mapsto \alpha(s)$ be a spacelike curve on the lightlike surface $M$ and $\{T, Y, Z\}$ is the Darboux frame along the curve $\alpha$ of the lightlike surface $M$. Then

$$
\kappa_{z}=\left\langle\alpha^{\prime \prime}, Y\right\rangle
$$

is called $z$-curvatures at $\alpha(s)$ of the Darboux frame.

Definition 2.3. Let $\alpha: I \rightarrow M \subset \mathbb{E}_{1}^{3}, s \mapsto \alpha(s)$ be a spacelike curve on the lightlike surface $M$ and $\{T, Y, Z\}$ is the Darboux frame along the curve $\alpha$ of the lightlike surface $M$. Then

$$
\kappa_{y}=\left\langle\alpha^{\prime \prime}, Z\right\rangle
$$

is called $y$-curvatures at $\alpha(s)$ of the Darboux frame.

Definition 2.4. Let $\alpha: I \rightarrow M \subset \mathbb{E}_{1}^{3}, s \mapsto \alpha(s)$ be a spacelike curve with arc length parameter $s$ on the lightlike surface $M$ and $\{T, Y, Z\}$ is the Darboux frame along the curve $\alpha$ of the lightlike surface $M$. Then

$$
\tau_{r}=\left\langle Z^{\prime}, Y\right\rangle
$$

is called torsion at $\alpha(s)$ of the Darboux frame.

$\alpha: I \rightarrow M \subset \mathbb{E}_{1}^{3}, s \mapsto \alpha(s)$ is a spacelike curve with arc length parameter $s$ on the lightlike surface $M$, then we call $\kappa_{z}, \kappa_{y}, \tau_{r}$ the Darboux frame curvatures of $\alpha$ on the lightlike surface $M$. 


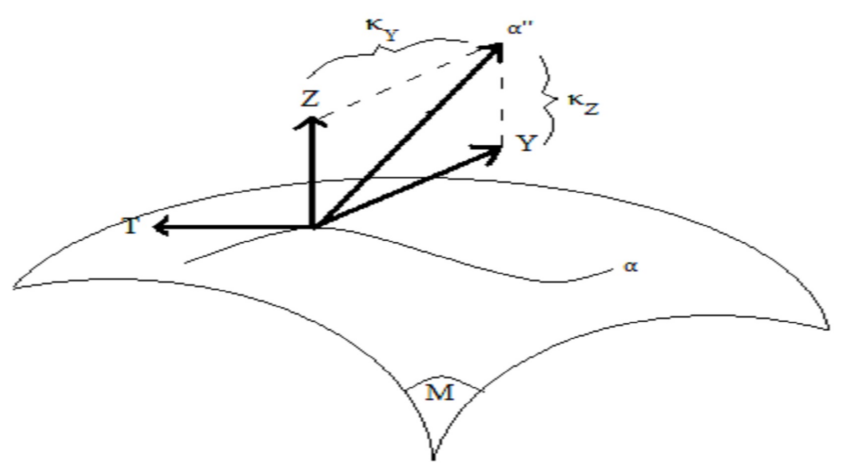

Figure 2. Darboux Frame $\{T, Y, Z\}$ and the frame curvatures $\kappa_{z}, \kappa_{y}, \tau_{r}$.

If we consider the normal vector $Z$ of the lightlike surface as $\alpha(s)$, then a particular case of the surface $M$ is obtained and it is a lighlike cone. The lightlike cone of $\mathbb{E}_{1}^{3}$, which is denoted by $Q^{2}$, is defined as the set of all lightlike vectors of $\mathbb{E}_{1}^{3}$, that is,

$$
\begin{aligned}
Q^{2} & =\left\{x \in \mathbb{E}_{1}^{3} \mid g(x, x)=0\right\} \\
& =\left\{x=\left(x_{1}, x_{2}, x_{3}\right) \in \mathbb{E}_{1}^{3} \mid x_{1}^{2}=x_{2}^{2}+x_{3}^{2}, x_{1} \neq 0\right\} .
\end{aligned}
$$

For the following part of the study, we inspired $[3,4]$

The generators of a lightlike cone are its normals. One of these generators of the lightlike cone is lying on the tangent plane of the cone. Moreover the other vectors on the tangent plane are spacelike vectors which are orthogonal to this generator.

Let $Q^{2}$ be the lightlike cone of $\mathbb{E}_{1}^{3}$ and $\alpha: I \rightarrow Q^{2} \subset \mathbb{E}_{1}^{3}, t \mapsto \alpha(t) \in Q^{2}$ be a regular curve in $Q^{2}$. If $\alpha(t)$ and $\alpha^{\prime}(t)$ are linearly independent at any $t \in I, \alpha^{\prime}(t)$ is spacelike. Then, if $\alpha(t)$ and $\alpha^{\prime}(t)$ are linearly independent, it can be parametrized by the arc length. Then the induced arc length $s$ of the curve $\alpha(t)$ can be defined by

$$
d s^{2}=\langle d \alpha(t), d \alpha(t)\rangle \text {. }
$$

From now on we assume that $\alpha$ is a unit spacelike curve, that is, $t(s)=\alpha^{\prime}(s)=\frac{d \alpha}{d s}$ is a spacelike unit tangent vector field of the curve $\alpha(s)$. $\alpha$ lies on the tangent plane of lightlike cone and it is also the normal vector field of the lightlike cone. Therefore, choosing the normal vector field of the lightlike cone $\mathbf{Z}=\alpha$ and a lightlike vector $\mathbf{Y}=\mathbf{y}$ which satisfy the conditions provided as follows,

$$
\begin{gathered}
\langle\alpha, \alpha\rangle=\langle y, y\rangle=\langle y, t\rangle=\langle\alpha, t\rangle=0 \\
\langle\alpha, y\rangle=\langle t, t\rangle=1 .
\end{gathered}
$$

The normal vector $Z$ of any lightlike surface is the position vector $\alpha$ of the lightlike cone. If we take the lightlike surface $Q^{2}$ instead of the surface $M$, then we get the Darboux frame of $Q^{2}$.

The purpose of the study is to investigate the Darboux frame of any lightlike surface, then we compare this frame with the asymptotic frame of lightlike cone which is defined by Liu (2004) [3]. Also, we examine the relationships between the curvatures of Darboux frame and the curvatures of the curve in $\mathbb{E}_{1}^{3}$,which is defined by Lopez (2008) [5]. Herefrom, similar relationships among the curvatures of lighlike cone and the curvatures of the curve in $\mathbb{E}_{1}^{3}$ will be .

The theorem, provided below shows that the derivatives of $T, Y, Z$ vector fields along $\alpha$ can be written according to the Darboux frame of $\alpha$.

Theorem 2.1. Let $\alpha: I \rightarrow M \subset \mathbb{E}_{1}^{3}, s \mapsto \alpha(s)$ be a curve with arc length on the lightlike surface $M$ and $\kappa_{z}, \kappa_{y}, \tau_{r}$ denote the Darboux frame curvatures of $\alpha$ on the lightlike surface $M$. Then,

$$
\left\{\begin{array}{c}
T^{\prime}=\kappa_{z} Z+\kappa_{y} Y \\
Z^{\prime}=-\kappa_{y} T+\tau_{r} Z \\
Y^{\prime}=-\kappa_{z} T-\tau_{r} Y
\end{array} .\right.
$$


$\kappa_{z}, \kappa_{y}, \tau_{r}: I \rightarrow \mathbb{R}$ are real valued smooth functions of the Darboux frame of $\alpha$. These functions depend on the curve $\alpha$ and the surface $M$. Hence $\kappa_{z}, \kappa_{y}, \tau_{r}$ depend on the position of the curve on the surface.

$\alpha: I \rightarrow M \subset \mathbb{E}_{1}^{3}, s \mapsto \alpha(s)$ is a curve on the lightlike surface $M . T(s)=\alpha^{\prime}(s)$ is to be unit tangent vector field of $\alpha$; then,

$$
\alpha^{\prime \prime}(s) \in S p\{Z(s), Y(s)\} .
$$

As a result, $\alpha^{\prime \prime}(s)$ is on the $\{Z(s), Y(s)\}$ - plane and also $T(s)$ is orthogonal to the plane $\{Z(s), Y(s)\}$. Thus, $\{Z(s), Y(s)\}$ becomes a timelike plane. Since there exist 3 different types vectors included in a timelike plane, $\alpha^{\prime \prime}(s)$ can be spacelike, timelike and lightlike.

Given a regular curve $\alpha$, we want to assign for any point of the curve an orthonormal base that describes the geometry of curve. This will be given by the Frenet trihedron. The variation of this base along the curve will give us the information how the curve is deformed into the ambient space.

Let $\alpha$ be a spacelike curve in $\mathbb{E}_{1}^{3}$ and $T(s)=\alpha^{\prime}(s)$ is denoted the tangent vector of $\alpha$. There are three possibilities for the Frenet thrihedron of the spacelike curve $\alpha$ in $\mathbb{E}_{1}^{3}$, depending on the casual character of $T^{\prime}(s)$ [5]. Also, $\{T, N, B\}$ denotes the Frenet trihedron of $\alpha$ spacelike curve in $\mathbb{E}_{1}^{3}$ and $\{T, Y, Z\}$ is the Darboux frame along the curve $\alpha$, for $\forall s \in I$ due to the vectors $N, B, Z, Y$ are orthogonal to $T$, these four vectors are planar. By denoting $\kappa$, $\tau, N$ and $B$ the curvature, the torsion, the principal normal and the binormal of the curve $\alpha(s)$ in $\mathbb{E}_{1}^{3}$, respectively, then, using the pseudo orthonormal frame on the curve $\alpha(s)$, we have the following results.

We investigate known curvatures of the curve according to the Darboux frame and relationships between these curvatures (relationships between the curvatures $\kappa, \tau$ and $\kappa_{z}, \kappa_{y}, \tau_{r}$ for three cases depending on the $T^{\prime}(s)=\alpha^{\prime \prime}(s)$. This relationships is given as follows.

Case 1. Let $\{T, N, B\}$ denotes the Frenet trihedron of $\alpha$ spacelike curve in $\mathbb{E}_{1}^{3},\{T, Z, Y\}$ be the Darboux frame along the curve $\alpha$ and the vector $T^{\prime}(s)=\alpha^{\prime \prime}(s)$ be spacelike:

Lemma 2.1. The vector $T^{\prime}(s)$ is spacelike. The vectors $N$ and $B$ are called the normal vector and binormal vector respectively also, the curvature of $\alpha$ is defined by $\kappa$; such that, $\kappa(s)=\left\|T^{\prime}(s)\right\|, N(s)=\frac{T^{\prime}(s)}{\kappa(s)}$ and $B(s)=T(s) \times N(s)$. The Frenet equations are

$$
\left[\begin{array}{l}
T^{\prime} \\
N^{\prime} \\
B^{\prime}
\end{array}\right]=\left[\begin{array}{ccc}
0 & \kappa & 0 \\
-\kappa & 0 & \tau \\
0 & \tau & 0
\end{array}\right]\left[\begin{array}{l}
T \\
N \\
B
\end{array}\right] .
$$

The torsion of $\alpha$ is defined by $\tau=-\left\langle N^{\prime}, B\right\rangle$ [5].

Theorem 2.2. Let $\alpha: I \rightarrow M \subset \mathbb{E}_{1}^{3}, s \mapsto \alpha(s)$ be the curve on the lightlike surface $M,\{T, Z, Y\}$ be the Darboux frame $\kappa_{z}, \kappa_{y}, \tau_{r}$ be Darboux frame curvatures. $T$ is to be unit tangent vector field of $\alpha$, if $\alpha^{\prime \prime}(s)=T^{\prime}(s)$ is spacelike, we get;

$$
\kappa=\sqrt{2 \kappa_{z} \kappa_{y}}
$$

and

$$
\sqrt{\frac{\kappa_{z}}{\kappa_{y}}}=e^{\int\left(\tau-\tau_{r}\right) d_{s}} \text { or } \sqrt{\frac{\kappa_{z}}{\kappa_{y}}}=e^{\int-\left(\tau+\tau_{r}\right) d_{s}}
$$

hold, where $\kappa_{y}, \kappa_{z} \neq 0, \kappa_{z} \kappa_{y}>0$.

Case 2. Let $\{T, N, B\}$ denotes the Frenet trihedron of $\alpha$ spacelike curve in $\mathbb{E}_{1}^{3},\{T, Z, Y\}$ be the Darboux frame along the curve $\alpha$ and the vector $T^{\prime}(s)=\alpha^{\prime \prime}(s)$ be timelike:

If we cosider the lightlike surface as a lightlike cone, we get following definitions and we can demonstrate the relations between Darboux frame of any lightlike surface and lightlike cone which is a special lightlike surface.

Definition 2.5. Let $\alpha: I \rightarrow Q^{2} \subset \mathbb{E}_{1}^{3}, s \mapsto \alpha(s)$ be a spacelike curve with arc length parameter $s$ on the lightlike cone, $t(s)=\alpha^{\prime}(s)$ be tangent vector field of $\alpha$, and $y, \alpha$ are the lightlike vectors which satisfy Eq. (2.10); then, $\{t, \alpha, y\}$ establishes a frame field along the curve $\alpha$. The frame is called the Darboux frame along the curve $\alpha$ of the lightlike cone $Q^{2}$. The frame

$$
\{t, \alpha, y\}
$$

is called the asymptotic frame on $\mathbb{E}_{1}^{3}$ along the curve $\alpha(s)$ in $Q^{2}$ by Liu in [3]. 
Definition 2.6. Let $\alpha: I \rightarrow Q^{2} \subset \mathbb{E}_{1}^{3}, s \mapsto \alpha(s)$ be a spacelike curve on the lightlike cone $Q^{2}$, and, $\{t, \alpha, y\}$ be the Darboux frame along the curve $\alpha$ of the lightlike cone $Q^{2}$. Then

$$
\kappa_{z}=\left\langle\alpha^{\prime \prime}, y\right\rangle
$$

is called the cone curvature of $\alpha(s)$ of the Darboux frame.

The Darboux frame curvatures $\kappa_{z}, \kappa_{y}, \tau_{r}$ of the lightlike surface $M$ will become,

$$
\begin{aligned}
\kappa_{z} & =\left\langle T^{\prime}, Y\right\rangle=\left\langle\alpha^{\prime \prime}, y\right\rangle \\
\kappa_{y} & =\left\langle T^{\prime}, Z\right\rangle=\left\langle\alpha^{\prime \prime}, \alpha\right\rangle=-1 \\
\tau_{r} & =\left\langle Z^{\prime}, Y\right\rangle=\left\langle\alpha^{\prime}, y\right\rangle=0
\end{aligned}
$$

if the surface $M$ is the lightlike cone $Q^{2}$.

The theorem, provided below shows that the derivatives of $t, \alpha, y$ vector fields along $\alpha$ can be written according to Darboux frame of $\alpha$.

Theorem 2.3. Let $\alpha: I \rightarrow Q^{2} \subset \mathbb{E}_{1}^{3}, s \mapsto \alpha(s)$ be the curve with arc length parameter $s$ on the lightlike cone $Q^{2}$ and $\kappa_{z}$ denote the cone curvature and $\{t, \alpha, y\}$ denotes the Darboux frame of $\alpha(s)$ in the lightlike cone. Then,

$$
\left\{\begin{array}{l}
t^{\prime}(s)=\kappa_{z}(s) \alpha(s)-y(s) \\
\alpha^{\prime}(s)=t(s) \\
y^{\prime}(s)=-\kappa_{z}(s) t(s)
\end{array} .\right.
$$

This frame is consistent with the frame obtained in [3,4] $T(s)=t(s)=\alpha^{\prime}(s)$ is to be unit tangent vector field of $\alpha$, then it is obvious that;

$$
\alpha^{\prime \prime}(s) \in S p\{\alpha(s), y(s)\} \text {. }
$$

As a result, $\alpha^{\prime \prime}(s)$ is on the $\{\alpha(s), y(s)\}$ - plane and also $t(s)$ is orthogonal to the plane $\{\alpha(s), y(s)\}$. Thus, $\{\alpha(s), y(s)\}$ becomes a timelike plane. Since there exist 3 different types vectors included in a timelike plane, $\alpha^{\prime \prime}(s)$ can be spacelike, timelike and lightlike.

Theorem 2.4. Let $\alpha: I \rightarrow Q^{2} \subset \mathbb{E}_{1}^{3}, s \mapsto \alpha(s)$ be the curve on the lightlike cone $Q^{2},\{t, \alpha, y\}$ be the Darboux frame, $\kappa_{z}$ be the cone curvature function. $T=t$ is to be unit tangent vector field of $\alpha$, if $\alpha^{\prime \prime}(s)=t^{\prime}(s)=T^{\prime}(s)$ is spacelike,

$$
\kappa(s)=\sqrt{-2 \kappa_{z}(s)}
$$

and

$$
\tau(s)=-\frac{1}{2} \frac{\kappa_{z}^{\prime}(s)}{\kappa_{z}(s)}=-\frac{\kappa^{\prime}(s)}{\kappa(s)}
$$

hold, where $\kappa_{z} \neq 0, \kappa_{z}<0$. If we put the Equations (2.11) on the Equations (2.8), (2.9), then the Equations (2.12), (2.13) are accuired.

Lemma 2.2. The vector $T^{\prime}(s)$ is timelike. The normal vector is $N(s)=\frac{T^{\prime}(s)}{\kappa(s)}$, where $\quad \kappa(s)=\sqrt{-\left\langle T^{\prime}(s), T^{\prime}(s)\right\rangle}$ is $B(s)=T(s) \times N(s)$. The vectors $N$ and $B$ are called the normal vector and binormal vector respectively. The curvature of $\alpha$ is defined by $\kappa$. The Frenet equations are

$$
\left[\begin{array}{l}
T^{\prime} \\
N^{\prime} \\
B^{\prime}
\end{array}\right]=\left[\begin{array}{lll}
0 & \kappa & 0 \\
\kappa & 0 & \tau \\
0 & \tau & 0
\end{array}\right]\left[\begin{array}{l}
T \\
N \\
B
\end{array}\right]
$$

The torsion of $\alpha$ is defined by $\tau=\left\langle N^{\prime}, B\right\rangle$ [5]. 
Theorem 2.5. Let $\alpha: I \rightarrow M \subset \mathbb{E}_{1}^{3}, s \mapsto \alpha(s)$ be the curve on the lightlike surface $M,\{T, Z, Y\}$ be the Darboux frame $\kappa_{z}, \kappa_{y}, \tau_{r}$ be Darboux frame curvatures. If $\alpha^{\prime \prime}(s)=T^{\prime}(s)$ is timelike, we have;

$$
\kappa=\sqrt{-2 \kappa_{z} \kappa_{y}}
$$

and

$$
\sqrt{-\frac{\kappa_{z}}{\kappa_{y}}}=e^{\int\left(\tau-\tau_{r}\right) d_{s}} \text { or } \sqrt{-\frac{\kappa_{z}}{\kappa_{y}}}=e^{\int-\left(\tau+\tau_{r}\right) d_{s}}
$$

where $\kappa_{y}, \kappa_{z} \neq 0, \kappa_{z} \kappa_{y}<0$.

Theorem 2.6. Let $\alpha: I \rightarrow Q^{2} \subset \mathbb{E}_{1}^{3}, s \mapsto \alpha(s)$ be the curve on the lightlike cone $Q^{2},\{t, \alpha, y\}$ be the Darboux frame, $\kappa_{z}$ be the cone curvature function. If $\alpha^{\prime \prime}(s)=t^{\prime}(s)=T^{\prime}(s)$ is timelike, we have;

$$
\kappa(s)=\sqrt{2 \kappa_{z}(s)}
$$

and

$$
\tau(s)=-\frac{1}{2} \frac{\kappa_{z}^{\prime}(s)}{\kappa_{z}(s)}=-\frac{\kappa^{\prime}(s)}{\kappa(s)}
$$

where $\kappa_{z} \neq 0, \kappa_{z}>0$. If we put the Equations (2.11) on the Equations (2.15), (2.16), then the Equations which are above are accuired.

Theorem 2.7. Let $\alpha: I \rightarrow M \subset \mathbb{E}_{1}^{3}, s \mapsto \alpha(s)$ be the curve with non-lightlike normal vector on the lightlike surface $M$. The curve $\alpha$ is a planar curve if and only if

$$
\begin{gathered}
\tau_{r}=0, \\
\kappa_{z}=\text { constant }, \\
\kappa_{y}=\text { constant } .
\end{gathered}
$$

Lemma 2.3. The vector $T^{\prime}(s)$ is lightlike. The normal vector is defined by $N(s)=T^{\prime}(s)$, which is linear independent with $T(s)$. Let $B$ be the unique lightlike vector such that $\langle N, B\rangle=1$ and orthogonal to $T$. The vector $B(s)$ is called the binormal vector of $\alpha$ at $s$. The Frenet equations are

$$
\left[\begin{array}{l}
T^{\prime} \\
N^{\prime} \\
B^{\prime}
\end{array}\right]=\left[\begin{array}{ccc}
0 & 1 & 0 \\
0 & \tau & 0 \\
-1 & 0 & -\tau
\end{array}\right]\left[\begin{array}{l}
T \\
N \\
B
\end{array}\right]
$$

The function $\tau$ is called the torsion of $\alpha$. There is not definition of the curvature of $\alpha$ [5].

Theorem 2.8. Let $\alpha: I \rightarrow M \subset \mathbb{E}_{1}^{3}, s \mapsto \alpha(s)$ be the curve on the lightlike surface $M,\{T, Z, Y\}$ be the Darboux frame $\kappa_{z}, \kappa_{y}, \tau_{r}$ be Darboux frame curvatures. $T$ is to be unit tangent vector field of $\alpha$, if $\alpha^{\prime \prime}(s)=T^{\prime}(s)$ is lightlike,

$$
\kappa_{z} \kappa_{y}=0
$$

we know that, in this case, there is not definition of the curvature $\kappa$ of $\alpha$.

Theorem 2.9. Let $\alpha: I \rightarrow Q^{2} \subset \mathbb{E}_{1}^{3}, s \mapsto \alpha(s)$ be the curve on the lightlike cone $Q^{2},\{t, \alpha, y\}$ be the Darboux frame, $\kappa_{z}$ be the cone curvature function. $T=t$ is to be unit tangent vector field of $\alpha$, if $\alpha^{\prime \prime}(s)=t^{\prime}(s)=T^{\prime}(s)$ is lightlike,

$$
\kappa_{z}=0
$$

we know that, in this case, there is not definition of the curvature $\kappa$ of $\alpha$.

Example 2.1. $\alpha(s)=\left(\frac{1}{2} s^{2}+\frac{1}{2}, s, \frac{1}{2} s^{2}-\frac{1}{2}\right)$ is a spacelike curve on the lightlike cone. It is easily seen $\{t, \alpha, y\}$ the Darboux frame vectors as follows:

$$
\begin{aligned}
t(s) & =(s, 1, s) \\
\alpha(s) & =\left(\frac{1}{2} s^{2}+\frac{1}{2}, s, \frac{1}{2} s^{2}-\frac{1}{2}\right) \\
y(s) & =(-1,0,-1) .
\end{aligned}
$$

The cone curvature function of the curve $\alpha$ is zero:

$$
\kappa_{z}(s)=\left\langle\alpha^{\prime \prime}, y\right\rangle=-\frac{1}{2}\left\langle\alpha^{\prime \prime}, \alpha^{\prime \prime}\right\rangle=0 .
$$




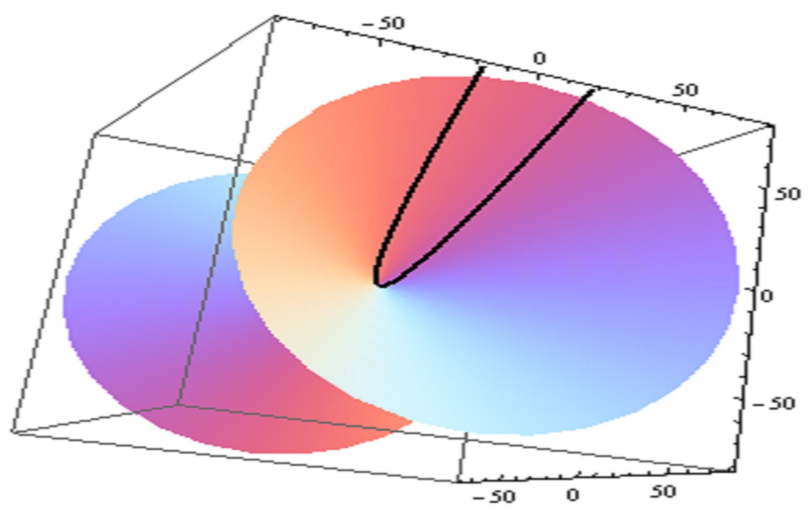

Figure 3. The cone curvature $\kappa_{z}$ is zero.

Theorem 2.10. The curve $\alpha: I \rightarrow Q^{2} \subset \mathbb{E}_{1}^{3}$ is planar curve if and only if the cone curvature function $\kappa_{z}$ of the Darboux frame is constant.

Definition 2.7. A helix in $\mathbb{E}_{1}^{3}$ is a regular curve such that $\langle T(s), v\rangle$ is a constant function for some fixed vector $v \neq 0$. Any line parallel this direction $v$ is called the axis of the helix [5].

Theorem 2.11. If a spacelike curve $\alpha$ in $\mathbb{E}_{1}^{3}$ is a helix, then $\frac{\tau}{\kappa}$ is a constant function. Also, let $\alpha$ be a spacelike curve with non-lightlike normal vector. If $\frac{\tau}{\kappa}$ is constant, then $\alpha$ is a helix [5].

Theorem 2.12. Let $\alpha: I \rightarrow Q^{2} \subset \mathbb{E}_{1}^{3}, s \mapsto \alpha(s)$ be the curve with non-lightlike normal vector on the lightlike cone $Q^{2}$, for some nonzero constant $c$ and the nonzero cone curvature function $\kappa_{z}$ satisfies the following condition

$$
\kappa_{z}^{\prime}(s)^{2}=8 c \kappa_{z}(s)^{3}
$$

if and only if $\alpha$ is a helix. We investigate this curvature for constant $c$ cases.

Conclusion 1. $\quad \alpha: I \rightarrow Q^{2} \subset \mathbb{E}_{1}^{3}, s \mapsto \alpha(s)$ is a helix curve on the lightlike cone $Q^{2}$, if considering $c=-1$, from the Eq. (2.23) we obtain

$$
\kappa_{z}^{\prime}(s)^{2}=-8 \kappa_{z}(s)^{3}
$$

Solving the Eq.(2.24),

$$
\kappa_{z}=-\frac{1}{2} s^{-2}
$$

is obtained.

Theorem 2.13. Let $\alpha: I \rightarrow Q^{2} \subset \mathbb{E}_{1}^{3}$ be a curve with arc length parameter s and the cone curvature function $\kappa_{z}=-\frac{1}{2} s^{-2}$, then $\alpha(s)$ can be written as follows:

$$
\alpha(s)=a_{1} s+a_{2} s \ln s+a_{3} s \ln ^{2} s
$$

where $a_{1}, a_{2}, a_{3} \in \mathbb{E}_{1}^{3} \quad$ [3]. Hence, we can give the following example.

Example 2.2. $\alpha(s)=s\left(\frac{1+\ln ^{2} s}{2}, \ln s, \frac{1-\ln ^{2} s}{2}\right)$ is a helix on the lightlike cone $Q^{2}$ in $\mathbb{E}_{1}^{3}$, with the axis $U=t-\frac{1}{2 s} \alpha+s y$. This curve's cone curvature function $\kappa_{z}$ is;

$$
\kappa_{z}=-\frac{1}{2 s^{2}}\langle(\ln s+1,1,-\ln s-1),(\ln s+1,1,-\ln s-1)\rangle=-\frac{1}{2} s^{-2} .
$$




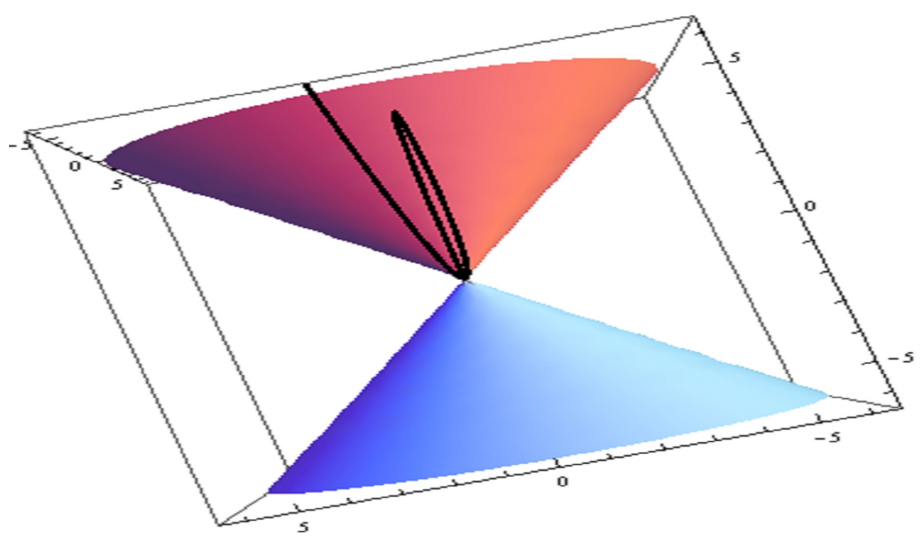

Figure 4. A helix on the light cone with lightlike axis.

Conclusion 2. $\quad \alpha: I \rightarrow Q^{2} \subset \mathbb{E}_{1}^{3}, s \rightarrow \alpha(s)$ is a helix curve on the lightlike cone $Q^{2}$, if considering $c>-1$, from the Eq. (25) we obtain

$$
\kappa_{z}^{\prime}(s)^{2}<-8 \kappa_{z}(s)^{3} .
$$

Solving the Eq.(2.26),

$$
\kappa_{z}>-\frac{1}{2} s^{-2}
$$

is obtained. Hence, we can give the following example:

Example 2.3. $\alpha(s)=\frac{s}{6}\left(s^{3}-s^{-3}, 2, s^{3}+s^{-3}\right)$ is a helix on the lightlike cone $Q^{2}$ in $\mathbb{E}_{1}^{3}$, with the axis $U=t+\frac{4}{s} \alpha+s y$. It is obvious that; the cone curvature function $\kappa_{z}$ of $\alpha$ is;

$$
\kappa_{z}=-\frac{1}{2}\left\langle\left(2 s^{2}-s^{-4}, 0,2 s^{2}+s^{-4}\right),\left(2 s^{2}-s^{-4}, 0,2 s^{2}+s^{-4}\right)\right\rangle=4 s^{-2} .
$$

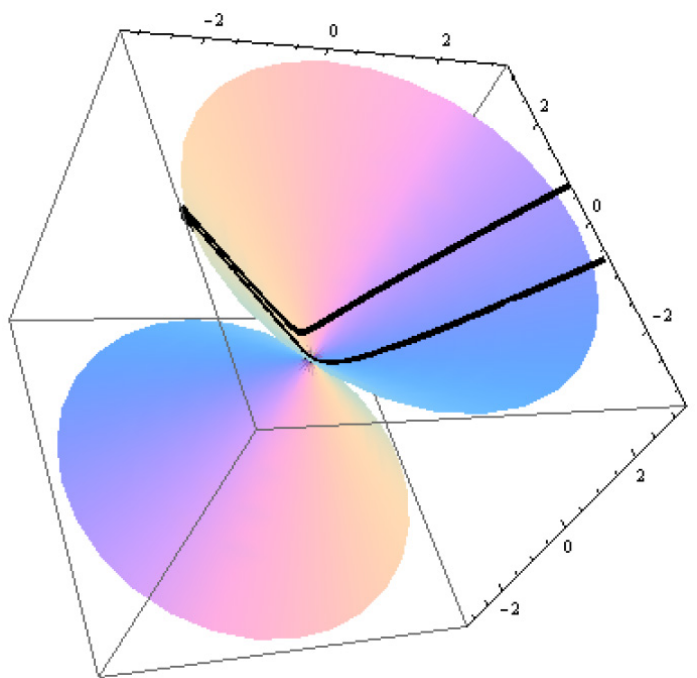

Figure 5. A helix on the light cone with spacelike axis.

Conclusion 3. $\quad \alpha: I \rightarrow Q^{2} \subset \mathbb{E}_{1}^{3}, s \rightarrow \alpha(s)$ is a helix curve on the lightlike cone $Q^{2}$, if considering $c<-1$, from the Eq. (25) we obtain

$$
\kappa_{z}^{\prime}(s)^{2}>-8 \kappa_{z}(s)^{3} .
$$

Solving the Eq. (2.27)

$$
\kappa_{z}<-\frac{1}{2} s^{-2}
$$


is obtained. Hence, we can give the following example:

Example 2.4. $\quad \alpha(s)=\frac{1}{\sqrt{7}}\left(s, s \cos \left(\sqrt{7} \ln \frac{s}{\sqrt{7}}\right), s \sin \left(\sqrt{7} \ln \frac{s}{\sqrt{7}}\right)\right)$ is a helix on the lightlike cone $Q^{2}$ in $\mathbb{E}_{1}^{3}$, with the axis $U=t-\frac{4}{s} \alpha+s y$. It is obvious that; the cone curvature function $\kappa_{z}$ of $\alpha$ is;

$$
\kappa_{z}=-\frac{1}{2}\left\langle\left(2 s^{2}-s^{-4}, 0,2 s^{2}+s^{-4}\right),\left(2 s^{2}-s^{-4}, 0,2 s^{2}+s^{-4}\right)\right\rangle=-4 s^{-2} .
$$

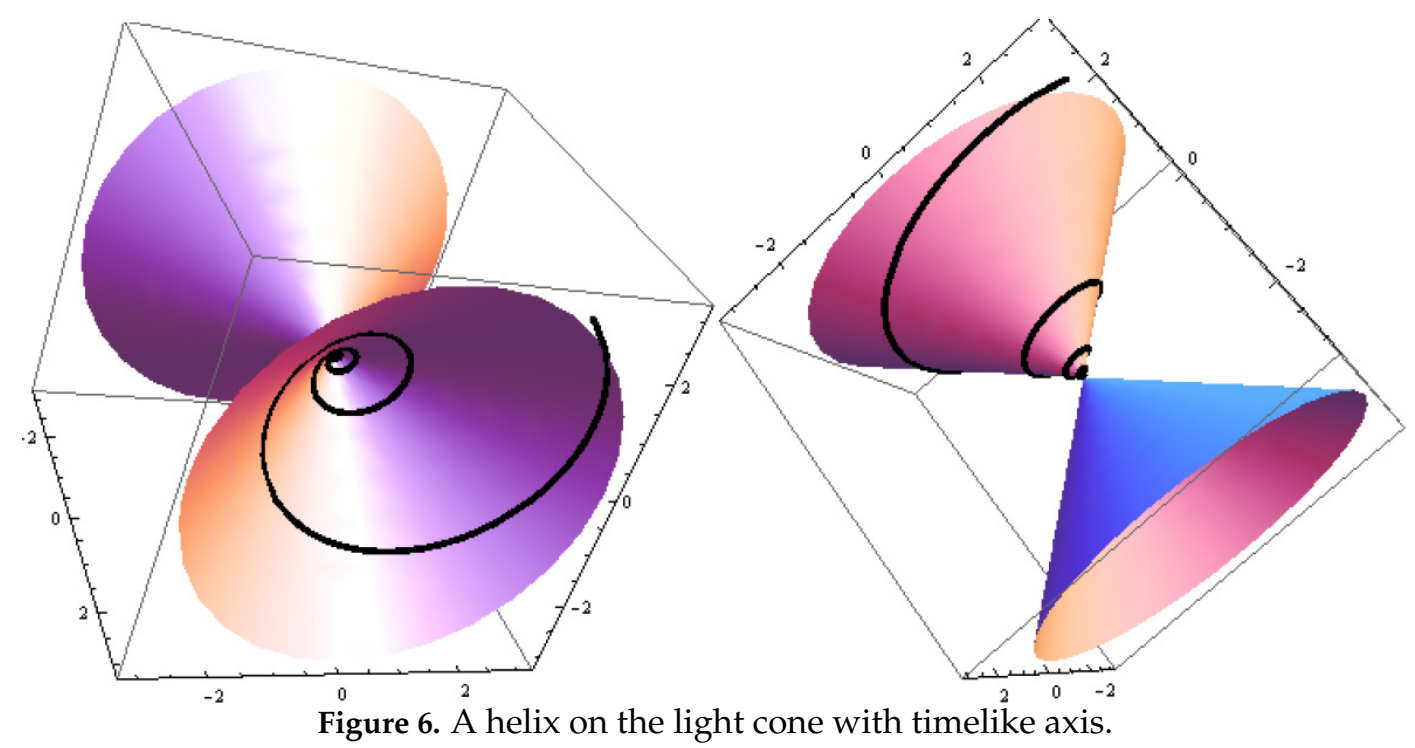

\section{References}

[1] Duggal, K., L., Bejancu, A., Lightlikesubmanifolds of Semi-Riemannian Manifolds andApplications, Kluwer Academic Publishers, Dordrecht, (1996).

[2] Duggal, K., L., Sahin, B., Differential Geometry of Lightlike Submanifolds, Birkhauser Verlag AG, Germany, (2010).

[3] Liu, H., Curves in the lightlike cone, Contributions to Algebra and Geometry, 45 (2004), No. 1, pp. 291-303.

[4] Liu, H., and Meng, Q., Representation Formulas of Curves in a Two- and Three-Dimensional Lightlike Cone, 59 (2011), pp. 437-451.

[5] Lopez, R., Diferential Geometry of Curves and Surfaces in Lorentz-Minkowski space, Mini-Course taught at the Instituto de Mathematica e Estatistica (IME-USP) University of Sao Paulo, Brasil (2008).

[6] O’Neill, B., Semi-Riemannian Geometry, Academic Press, NewYork 1983. .Zbl 0531.53051 (1983).

[7] Sabuncuoğlu, A., Diferensiyel Geometri, Nobel Yayıncılık, Ankara. (2011)

\section{Affiliations}


İSMAIL GÖK

AdDREsS: Ankara University, Dept. of Mathematics, 06100, Ankara-Turkey. E-MAIL: igok@science.ankara.edu.tr

NEJAT EKMEKCİ

AdDRESS: Ankara University, Dept. of Mathematics, 06100, Ankara-Turkey.

E-MAIL: ekmekci@science.ankara.edu.tr

YUSUF YAYLI

AdDress: Ankara University, Dept. of Mathematics, 06100, Ankara-Turkey.

E-MAIL: yayli@science.ankara.edu.tr 